\title{
SAMIA KASSAB-CHARFI, ADEL KHEDHER, Un siècle de littérature en Tunisie. 1900-2017
}

\section{Yves Chemla}

\section{(2) OpenEdition}

\section{Journals}

\section{Édition électronique}

URL : https://journals.openedition.org/studifrancesi/23299

DOI : $10.4000 /$ studifrancesi.23299

ISSN : 2427-5856

\section{Éditeur}

Rosenberg \& Sellier

\section{Édition imprimée}

Date de publication : 1 avril 2020

Pagination : 225-227

ISSN : 0039-2944

\section{Référence électronique}

Yves Chemla, "Samia kASSAB-CharfI, Adel kHEdher, Un siècle de littérature en Tunisie. 1900-2017 》, Studi Francesi [En ligne], 190 (LXIV | I) | 2020, mis en ligne le 01 mai 2020, consulté le 02 août 2021. URL http://journals.openedition.org/studifrancesi/23299; DOI : https://doi.org/10.4000/studifrancesi. 23299

Ce document a été généré automatiquement le 2 août 2021.

\section{(c) (i) ()}

Studi Francesi è distribuita con Licenza Creative Commons Attribuzione - Non commerciale - Non opere derivate 4.0 Internazionale. 


\title{
SAMIA KASSAB-CHARFI, ADEL KHEDHER, Un siècle de littérature en Tunisie. 1900-2017
}

\author{
Yves Chemla
}

\section{RÉFÉRENCE}

SAMIA KASSAB-CHARFI, ADEL KHEDHER, Un siècle de littérature en Tunisie. 1900-2017, Paris, Champion, 2019, 550 pp.

1 Assurément, l'ouvrage publié écrit par Samia Kassab-Charfi et Adel Khedher est important à deux titres, au moins. D'abord, parce qu'il révèle la richesse et la profondeur d'une littérature qui reste largement méconnue dans les espaces universitaires; ensuite parce qu'il en dresse une vue à la fois perspective et rythmée par des études de détail. Cet ouvrage est un manuel érudit: bibliographie, index et sommaire permettent un accès efficace aux informations recherchées, tout en engageant à la circulation, ce qui est sans doute le propre des ouvrages appelés à devenir une référence. Manuel érudit, il l'est encore par les considérations méthodologiques et théoriques qui sont particulièrement fréquentes.

2 Un Siècle de littérature tunisienne 1900-2017 reprend et prolonge les études menées par des chercheurs tels le poète Tahar Bekri ou Jean Fontaine, dont l'Histoire de la littérature tunisienne a fait date. Cependant, si ces travaux ainsi que ceux de Charles Bonn et de Guy Dugas sont aisément accessibles, ils font la part belle aux textes d'expression française, qui est d'abord, il faut le rappeler, leur objet de prédilection. La lecture de ce livre révèle à qui en douterait que la littérature tunisienne est d'abord de langue tunisienne, mais qu'elle manifeste un singulier tropisme pour le contact des langues.

3 L'organisation de l'ouvrage dit l'enroulement des langues: après une traversée de la genèse historique, assez grandiose, il faut le reconnaître, puisqu'elle démarre des traces puniques et couvre jusqu'au XIX ${ }^{\mathrm{e}}$ siècle, l'ouvrage mène une étude fine des différentes 
littératures, dans les différentes langues d'écriture et de lecture. Tour à tour punique, romaine, kabyle, chrétienne, juive, islamique, hispano-mauresque, espagnole, ottomane... cette région a vu les traditions se mêler, les récits s'enrouler les uns dans les autres. À partir de ce rappel, les auteurs étudient successivement, et pas nécessairement dans un ordre chronologique, ne sacrifiant justement pas la cohérence logique de leur étude au seul parcours temporel, la poésie de langue arabe, les écrivains sardes, italiens et français, la poésie d'expression française, la nouvelle de langue arabe, pour laquelle les écrivains tunisiens ont déployé un talent reconnu, le roman de langue arabe puis la nouvelle et le roman francophones. La littérature tunisienne du dehors fait l'objet d'un chapitre, suivi du théâtre et de l'essai. Une anthologie de textes traduits et de textes écrits en français complète le parcours de cette étude particulièrement éclairante.

L'étude de la littérature de langue arabe montre comment celle-ci s'est détachée de la tradition et combien celle-ci contraignait l'avènement d'une modernité à laquelle le mouvement de la Nahda, au XIX ${ }^{\mathrm{e}}$ siècle, en appelait pourtant. Dans la poésie, les formes produites dans la langue dialectale, par opposition à celle de la langue classique, revendiquent en quelque sorte droit de cité. La figure centrale de Chebbi rayonne sur cette période. Dans une conférence donnée en 1929 et restée célèbre, il déclare ainsi que l'ancienne poésie n'a été créée que "pour des cœurs que figea l'immobilisme de la mort» (p. 51). Cette déclaration peut presque être considérée comme le prolégomène des lettres tunisiennes $\mathrm{du} \mathrm{xx}^{\mathrm{e}}$ siècle. Mais aussi dans ces années et jusqu'à l'indépendance, en 1956, et même après, les poètes célèbrent aussi l'amour patriotique. Les désillusions qui ont succédé aux «soleils des indépendances» feront glisser en partie ces thèmes vers le désenchantement, et l'écriture vers l'expression sarcastique et l'autodérision. Ce faisant ils modifient considérablement l'écriture poétique. D'abord en prenant délibérément parti pour le choix de la langue vernaculaire entendue comme la langue du quotidien; ensuite en décidant d'abandonner définitivement les formes classiques, voire en adoptant la forme du vers libre et du poème en prose.

5 Dans le premier tiers du siècle, ce sont aussi des écrivains d'origine italienne ou française qui émergent. La figure de Mario Scalesi en est sans doute la plus connue: poète maudit, difforme, survivant dans une Tunisie populaire dont la majorité pauvre est meurtrie par des fléaux tels la faim, la maladie, la déshérence. Sa poésie est tout entière dans l'entrebâillement entre deux extrêmes: les miasmes de l'enfermement sépulcral et les envolées solaires. Tous les Poèmes d'un Maudit, ouvrage composé par des membres de la Société des Écrivains d'Afrique du nord, après sa mort prématurée à Palerme, en 1922, seul et abandonné, son corps jeté à la fosse commune, constituent autant de jalons d'une œuvre pré-posthume, hantée par l'espérance et surtout son inverse, qui prend le pas, et conduit à des circulations mortifères entre les images, les lieux et les époques. Mais aussi, autre versant du personnage, il a endossé une identité nord-africaine, et le regard plein d'acuité qu'il porte sur son univers de référence le conduit à considérer de façon critique l'exotisme en vogue, en particulier dans la littérature coloniale.

D'autres veines prennent peu à peu leur appui. Ce sont les jeux sur les parlures populaires avec Kaddour Ben Nitram, qui constituent les langues arabes et française comme des langues «cassées» et pourtant compréhensibles, non sans doute dans le cadre d'une complaisance à la limite du préjugé, mais bien comme la prise en considération de la déshérence populaire en matière d'éducation: la nouvelle ou le 
sketch tournent en dérision les manières hautaines de parler et une certaine façon d'écrire des histoires, comme de se tenir à distance des miasmes du populaire. Cesare Luccio (1906-1980) se fait le chroniqueur de la communauté sarde de Tunisie, à cheval lui-même sur un double statut pas complètement assumé: à la fois colonisé, mais aussi quelque peu du côté des colonisateurs. La plupart des écrivains de cette période sont ainsi partagés, à l'exception notoire de Francesco Cucca (1882-1947), écrivain anarchiste, pourfendeur de la mainmise coloniale sur le pays. C'est le cas d'un Guido Medina (1888-1967), par exemple, qui publie ses poèmes en langue italienne, dans des formes souvent classiques, comme le sonnet. Il s'y livre à des recherches formelles, comme dans Poemetti al vento (Firenze, Felice Le Monnier, 1937) ou Poema del sole (Paris, Parisis Édition, 1939), inspirés par la poésie de Valéry, qu'il rencontre à Tunis. Mais aussi, Medina élève une protestation véhémente après la promulgation des lois raciales en Italie, après avoir cru en la sincérité mussolinienne. Il Grido d'un Italiano (Tunis, Imprimerie Hadida, s. d. [1938]) est presque immédiatement traduit à Paris (Parisis Édition, 1939) et connaîtra une certaine renommée. Il rejette une nation qui a cédé sur tous les plans, alors que les déclarations affirmaient au contraire la possibilité d'une renaissance. Désormais, il écrit et publie en français.

7 Ce sont aussi dans ces années les premières plumes judéo-tunisiennes qui émergent. Les Juifs de Tunisie, après avoir produit une littérature en judéo-arabe, se sont emparé de la langue française. Daisy Sebag ( $x^{\mathrm{e}}$ siècle), Ryvel (1898-1972), Jacques Véhel ( $\mathrm{xx}^{\mathrm{e}}$ siècle), Vitalis Danon (1898-1969) tracent les contours de cette présence qui va aller s'effaçant après la fin de la guerre.

8 La poésie d'expression française connaît en revanche un essor qui ne se dément pas. Abderrazak Bannour a depuis longtemps étudié les linéaments de cette écriture, qui connaît aussi une vitalité avérée: Hedi Bouraoui, Mohamed Aziza, mais aussi Majid El Houssi (1948-2001), ce dernier installé à Padoue, où il enseignait à l'université, inscrivent des thématiques modernes dans leur écriture: le voyage, l'exil, la berbérité. Une longue étude consacrée à Majid El Houssi montre combien est féconde la rencontre et le souci de l'altérité qui en est la conséquence la plus directe. D'autres œuvres majeures sont analysées comme celles de Tahar Bekri et Moncef Ghachem. Les productions les plus récentes sont abordées avec Slaheddine Haddad, Amina Saïd, Moëz Majed ou Aymen Hacen.

9 Le chapitre sur la nouvelle de langue arabe est central dans l'ouvrage: l'art de la nouvelle a été un des fils conducteurs de cette littérature pendant le $\mathrm{xx}^{\mathrm{e}}$ siècle, et le demeure assurément. On compte en effet plus de 400 recueils publiés entre 1956 et 2010, sans compter les publications en revues. Articulant le récit et l'analyse de types variables d'expériences humaines, ce qui tisse l'entrelacs des nouvelles transmises par la presse dès l'origine de cette dernière, justement, leur étude est d'autant plus efficace qu'elle analyse les différents types de protagonistes qu'elles mettent en scène. L'étude vaut presque comme monographie: les auteurs étudient les œuvres des écrivains initiaux comme Sadok Rezgui (1874-1939), Slimane Jedoui (1881-1951) et Mohamed Habib (1902-1980). Dans l'entre-deux guerres le groupe de Taht Essour, du nom d'un café sous les remparts développe encore le genre, l'amplifie par la création de revues. Dans les générations actives après la fin de la deuxième guerre, ce qui importe est un réalisme descriptif «qui se préoccupe plus de l'expression la plus exacte du vécu terre-à-terre» et qui parvienne à rendre compte de «la conscience malheureuse des individus» (160). Béchir Khraïef (1917-1983) montre comment l'ancien monde a vacillé devant l'avancée 
de la modernisation, en particulier pour ce qui est de la place des femmes. Peu à peu, l'art de la nouvelle va subir un désenchantement comparable à celui que connait la poésie à la même époque post-indépendance. La nouvelle va glisser dans des thématiques oniriques, ou bien dans un réalisme quasi-magique, dont la dimension de critique sociale va s'amplifiant avec le temps. Le dérèglement psychique fait ainsi son entrée dans les thématiques, comme une charge contre les veuleries du quotidien, contre les tabous multiples qui enferment les sociétés dans la reproduction du mal.

10 Le chapitre sur le roman de langue arabe suit le même schéma d'analyse. Après une étude des premiers d'entre eux, en particulier le Périple à travers les bars de la Méditerranée (1935) d'Ali Douagi (1909-1949), les auteurs montrent qu'à partir de 1956 le roman tunisien voit croître ses formes, en particulier par la prise en charge des mutations des mœurs. Peu à peu, les écrivains tels Mustapha Fersi, Brahim Darghouti ou Mustapha Kilani vont prendre conscience que le roman «est en quête d'une connaissance qu'il n'est susceptible de recueillir qu'une fois l'écriture achevée» (p. 207). Les thématiques se resserrent autour de perspectives critiques en particulier politiques. Avec Leïla Mami (1944), les histoires racontent la résistance des femmes au joug du patriarcat.

11 Écrire en français a été un des leviers de sédition contre la tradition. Salah Garmadi (1933-1982), Rafik Ben Salah (1948, installé en Suisse, comme Fawzi Mellah, 1946), placent la critique de mœurs au cœur du dispositif romanesque. Les romancières Jalila Hafsia, Sophie El Goulli, Azza Filali, Fawzia Zouari mêlent à cette critique l'analyse du creuset identitaire qu'a toujours été la Tunisie, ou qu'elle croit être, et la dénonciation des forces obscurantistes et prédatrices qui dépossèdent le pays de son âme: de son énergie, de ses dynamiques et de ses forces vives, comme dans Ouatann de A. Filali (Tunis, Elyzad, 2012).

12 Une autre dimension de cette littérature est traitée, qui permet là encore d'outrepasser les frontières trop souvent de mise dans l'étude des littératures nationales: les écrivains à la fois du dedans et du dehors selon le mot de l'une d'entre eux, Sophie Bessis (1947): Albert Memmi (1920), ses frères, Georges (1929) et Max (1935), Gisèle Halimi (1927), Marco Koskas (1951), Nine Moati (1938), Annie Goldmann (1931), et d'autres encore, constituent cette pléiade d'auteurs qui remettent en cause les verrouillage des frontières. Ainsi Adrien Salmieri (1929) et Sicca Venier (1922-2005), rappellent la mémoire de la présence italienne en Tunisie. Enfin, Hélé Béji (1948) et Abdelwahab Meddeb (1946-2014) repensent les postures sociales et politiques liées aux dimensions spirituelles et culturelles, souvent défigurée par l'intransigeance religieuse et le nationalisme étroit.

13 Les deux chapitres qui terminent l'étude, l'un consacré au théâtre et l'autre à l'essai, complètent l'analyse des richesses des corpus. Ils confirment la place exceptionnelle de ces littératures tunisiennes, dont le caractère novateur voire révolutionnaire dans le monde arabe est si particulier qu'il en provoque, on le sait, certaines réticences dans les autres capitales culturelles du monde arabe. En effet, et on peut généraliser ce que Samia Kassab-Charfi et Adel Khedher affirment de l'essai: cette littérature devrait être considérée comme une des «armes miraculeuses» qui aident à «combattre l'inhibition inhérente aux coercitions du dogme» (393).

14 On ajoutera un tout dernier mot. L'ouvrage, qui peut se lire d'un trait, accomplit ce que tout manuel devrait se donner comme règle d'écriture et comme horizon d'attente: susciter réellement le désir de lire les ouvrages qu'il présente. 